\title{
Progress on DNA Barcoding of Fungi
}

\section{CONSORTIUM FOR THE BARCODE OF LIFE}

On 17-18 April 2011, members of the Fungal Working Group (FWG) of the Consortium for the Barcode of Life (CBOL) met in the classic surroundings of the King's Chamber (Koningszaal), of the Bazel Conference Centre, of the Amsterdam City Archive. Before the meeting, an international group of more than 75 mycologists from 18 countries contributed sequences or DNA to the effort, with a concerted attempt to obtain representative sequences for all major lineages of the Eumycota. Four markers were selected for comparison, three regions of the nuclear ribosomal DNA and one protein coding gene. Of the rDNA markers, the internal transcribed spacer (ITS) was the main focus because of its broad utility as a species marker in taxonomic and ecological studies. The large subunit (LSU) was selected because of its similar application in some groups, in particular yeasts. The small subunit (SSU) was included in an attempt to demonstrate to bacteriologists, who use the $16 \mathrm{~S}$ as a de facto barcode, that the region is unsuitable for species identification in fungi. The largest subunit of ribosomal polymerase II (RPBI) was selected as a representative protein coding gene because of the general good PCR success with this gene and its utility as phylogenetic marker in the AFTOL project. For some fungi, the second largest subunit of ribosomal polymerase II (RPB2) and another recently introduced marker $M C M 7$, also utilized in the second AFTOL project, were sequenced. The sequences were either generated in the participants' laboratories, or through a collaboration with Elena Bolchacova at the LifeTech facilities in California. The data were assembled in a custom-made database implemented by Vincent Robert (CBS-KNAW Fungal Biodiversity Centre, Utrecht) using the BioloMICS database package, and then analysed in a manner similar to that used by the Plant DNA barcoding consortium by John Spouge.

For much of the first day, participants gave short presentations focusing on a broad range of taxonomic groups, considering the newly generated data in the context of previous results. In general, there was a clear preference for the ITS as a species barcode across all groups, although RPB1 often produced more resolving power. ITS has lower resolution in some groups, but the overall identification success rate was calculated at $72 \%$ for all fungi, similar or slightly superior to the success rate of the two barcode system adopted for plants. The high PCR success rate is a critical aspect of the possible utility of the ITS as a barcode. Although the identification success for RPB1 was higher at $81 \%$, the much lower PCR success rate is a serious limitation. Almost no data could be generated for the basal fungal lineages and the only data set generated for those groups came from DNA samples sent to LifeTech, where M13 sequencing was used. Although the utility of other protein-coding genes were discussed at the meeting, in particular translation elongation factor 1- $\alpha$, most participants acknowledged that while these markers would probably have a higher identification success rate than ITS, that PCR success rates would be too low, at least with current primers, to seriously consider any of them as fungal barcodes. This was emphasized by researchers working on environmental sequences, who preferentially use ribosomal loci. In general the SSU had the lowest species identification success rate, and it was noted that the primers used amplify algal sequences preferentially in lichenized samples. The LSU was intermediate, behind ITS and RPB1.

On the second day, there was a careful discussion of whether a two gene barcode system should be considered, especially for yeasts, with both the ITS and LSU being used. One critical consideration for a twogene system is balancing increased sequencing costs versus the expected benefit of the second barcode. During barcode database development, both markers would have to be sequenced. Although it is clear that LSU

is superior to the ITS for recognizing species in some groups of yeasts, a combined LSU/ ITS system gave only a modest increase in identification success. With the assent of the yeast taxonomists participating in the meeting, we agreed that it would be preferable to propose a one gene barcoding system for the fungi, and that we would recommend that this be the ITS. Consideration of a second barcode gene, especially for the Ascomycota, will continue for the next 2-3 years as genome mining efforts continue.

The meeting concluded with the establishment of a plan to prepare a publication based on the new data set, to be used as a supporting document for the formal proposal to CBOL for fungal DNA barcoding. From the concluding remarks of the meeting, it is clear that mycologists are interested in participating in the DNA barcoding movement, with projects on quarantine-relevant fungi, medicallyimportant fungi and indoor moulds already underway. Serious interest was also expressed in sequencing fungal herbarium specimens, and pursuing an international research coordination network (perhaps affiliated with IBOL, the International Barcode of Life network) for fungal DNA barcoding.

The Fungal Working group will convene again in Adelaide, Australia, for the $4^{\text {th }}$ CBOL conference on DNA Barcoding, scheduled for 28 November to 3 December 2011. The call for abstracts is now open $^{1}$. There are three categories of sessions plus poster presentations: plenary sessions, taxon-oriented parallel technical sessions, and thematic parallel technical sessions. It is foreseen that the meeting with have a strong representation from mycologists, plant pathologists and microbiologists working with fungi.

We are very grateful to David Schindel and CBOL for their financial support of the Amsterdam meeting, and to Lorenzo Lombard, Rob Samson, and others at the CBSKNAW Fungal Biodiversity Centre for doing an excellent job in preparing the venue and accommodations in Amsterdam.

Conrad Schoch, Keith Seifert and Pedro Crous (schoch2@ncbi.nlm.nih.gov) 


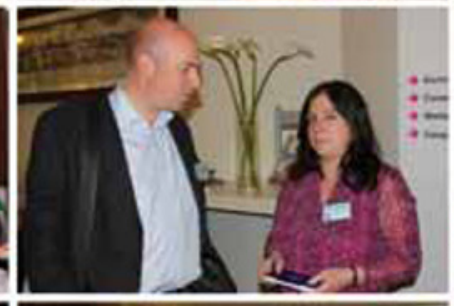

\section{-}

8f
I. of
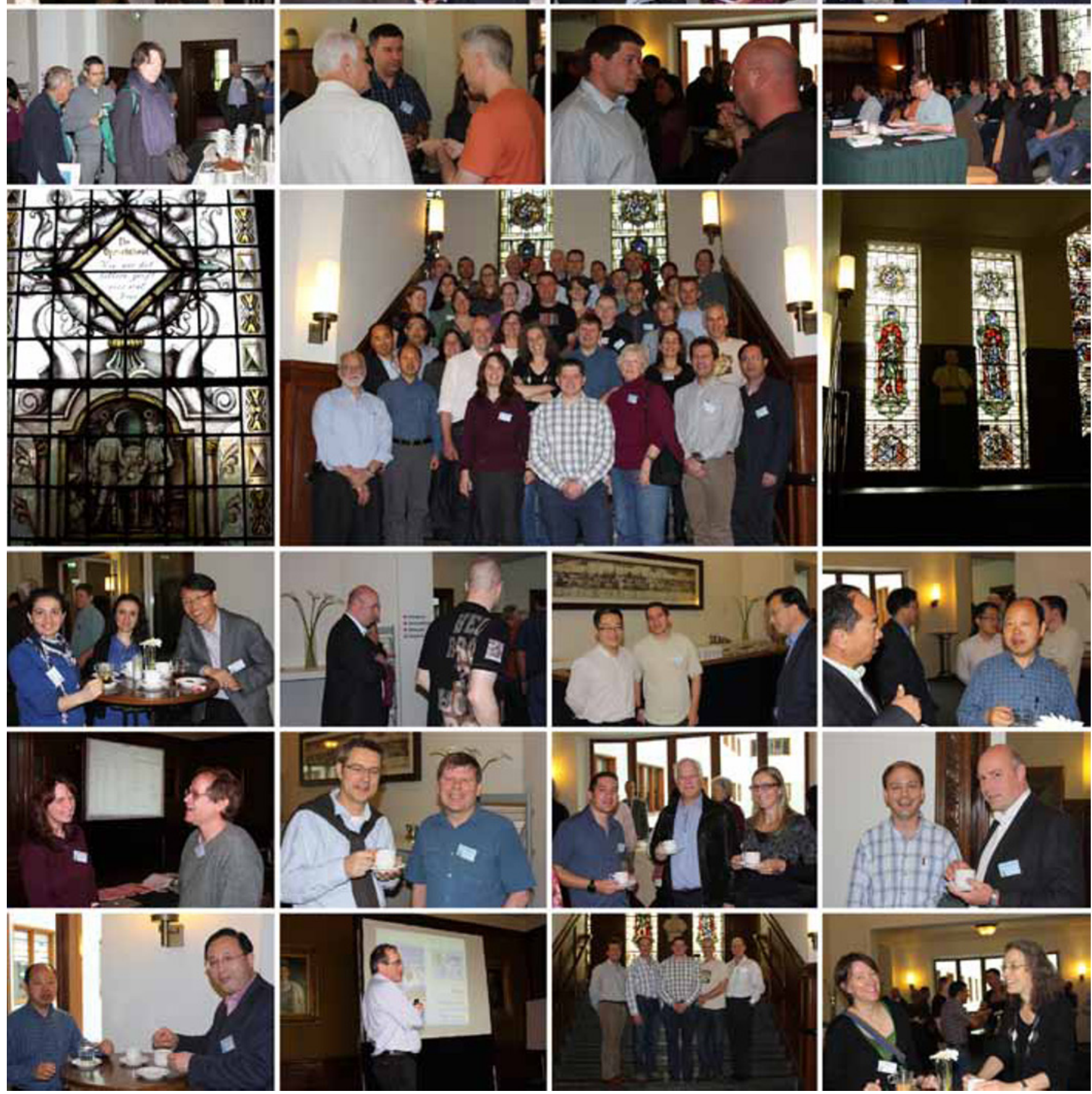

Scenes from the fungal barcoding meeting held in the Koningszaal of the Bazel Conference Centre, Amsterdam, on 17-18 April 2011. 


\section{CBS Symposium: One Fungus = One Name}

\section{On 19-20 April 2011, the CBS-KNAW} Fungal Biodiversity Centre, under the auspices of the International Commission on the Taxonomy of Fungi (ICTF), organized a symposium on 'One Fungus = One Name' at the Trippenhuis (home of the Royal Netherlands Academy of Arts and Sciences) in Amsterdam. Ninety participants from 23 countries attended the symposium.

The programme consisted of 18 presentations, which discussed various important nomenclatorial issues. Questions which were debated, included:

(1) Fungi are the only kingdom where single species are allowed to have more than one valid scientific name - is this solution to dealing with fungal pleomorphy still appropriate?

(2) Does DNA sequencing make dual nomenclature superfluous?

(3) Can the International Botanical Code be modified to enable this process, or would a MycoCode be more effective?

(4) How can the mycological community get rid of the legacy of dual nomenclature and Article 59 without nomenclatural chaos?
Important contributions were presented by John Taylor, Mike Wingfield, David Hawksworth, Walter Gams, Keith Seifert, Scot Redhead, Uwe Braun, Andre Levesque, Ulf Thrane, Sybren de Hoog, Wieland Meyer, Janos Varga, Jos Houbraken, FengYan Bai, and Joost Stalpers, which provoked a lively discussion. On the second day, David Hawksworth prepared a document, based on a draft written by Keith Seifert, and entitled the Amsterdam Declaration on Fungal Nomenclature. The wording paper was discussed in detail and accepted by almost all of the audience. After the symposium, the Amsterdam Declaration was circulated to participants and more widely, and the final edited version is published in this issue of IMA Fungus (pp. 105-112).

During the symposium, a festive reception was given to celebrate that Rob Samson, Joost Stalpers, and Sybren de Hoog had each conducted mycological research for 40 years at the CBS-KNAW Fungal Biodiversity Centre -120 years of mycological research! On 20April, two important CBS awards were made. The Westerdijk Award was presented to Jack Fell (Miami) for his contribution of many yeast cultures to the CBS collection during his career, while David Hawksworth (Madrid and London) received the Joseph von Arx Award for his excellent career contribution to fungal

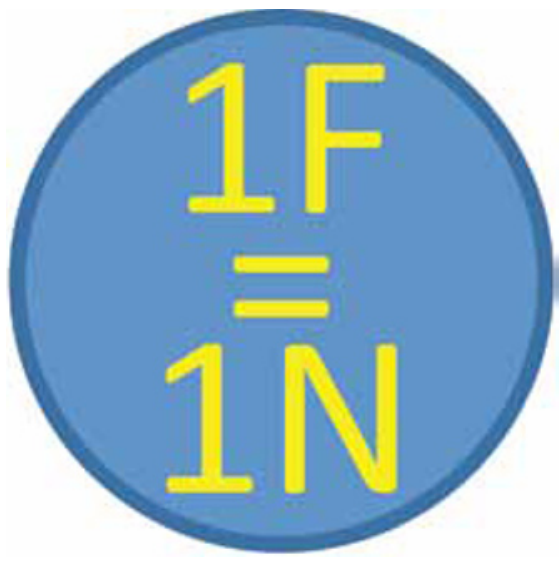

systematics (see pp. (10)-(11) in this issue). On the same day, two important books launched and copies presented to the Science Director of the Academy, Theo Mulder. The first book, The Yeasts - a taxonomic study was presented by all three authors, Clete Kurtzman, Jack Fell, and Teun Boekhout; and the second book, The Genera of Hyphomycetes by Keith Seifert and Walter Gams (also on behalf of their co-authors Bryce Kendrick and Gareth Morgan-Jones who could not be present). Reviews of these two landmark works are included later in this issue (pp. (30)-(31)).

Pedro W. Crous

(p.crous@cbs.knaw.nl)

\section{Australian Plant Pathology Society Workshop}

The Australasian Plant Pathology Society (APPS) held a 2-day post-conference workshop on the 'Identification of some common genera of plant associated anamorphic Ascomycetes' in one of the Biological Sciences teaching laboratories at Charles Darwin University in Darwin, Queensland, Australia on 30 April-1 May 2011. The workshop was attended by about 30 participants, mostly plant pathologists from universities and government agencies (especially those concerned with biosecurity) in Australia. The first day was spent looking at fresh and dried specimens of anamorphic Mycosphaerellaceae and the next day at cultures of alternarioid, helminthosporioid, and hypocrealean fungi. The course was turoured by Elaine Davison, Ian Pascoe, Pedro Crous, and Roger Shivas, with considerable help from Thomas Marney who generated and organised the specimens and cultures.

Pedro W. Crous

(p.crous@cbs.knaw.nl) 


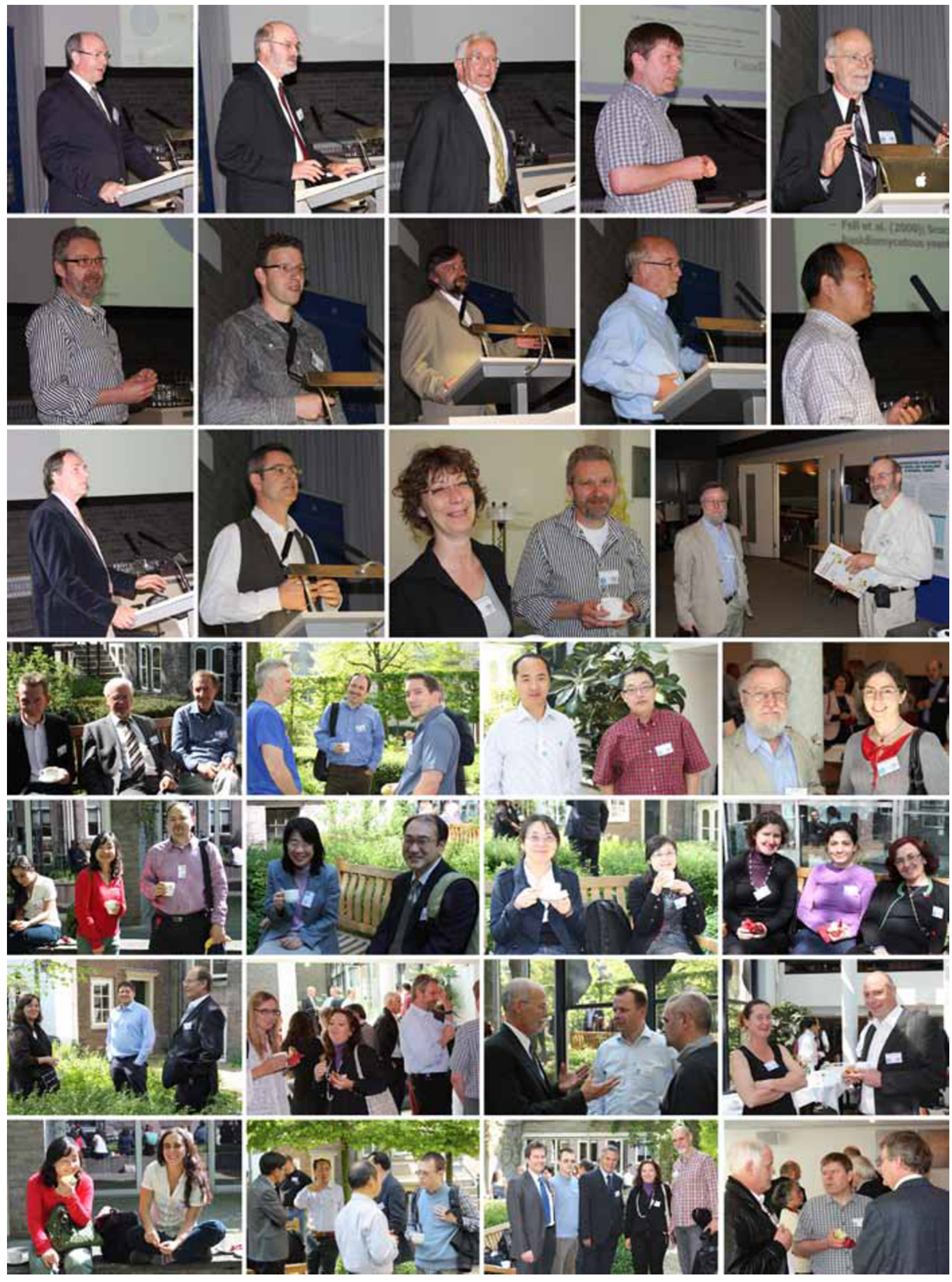

Scenes from the One Fungus = One Name symposium held in the Trippenhuis, headquarters of the Royal Netherlands Academy of Arts and Sciences, Amsterdam, on 19-20 April 2011 


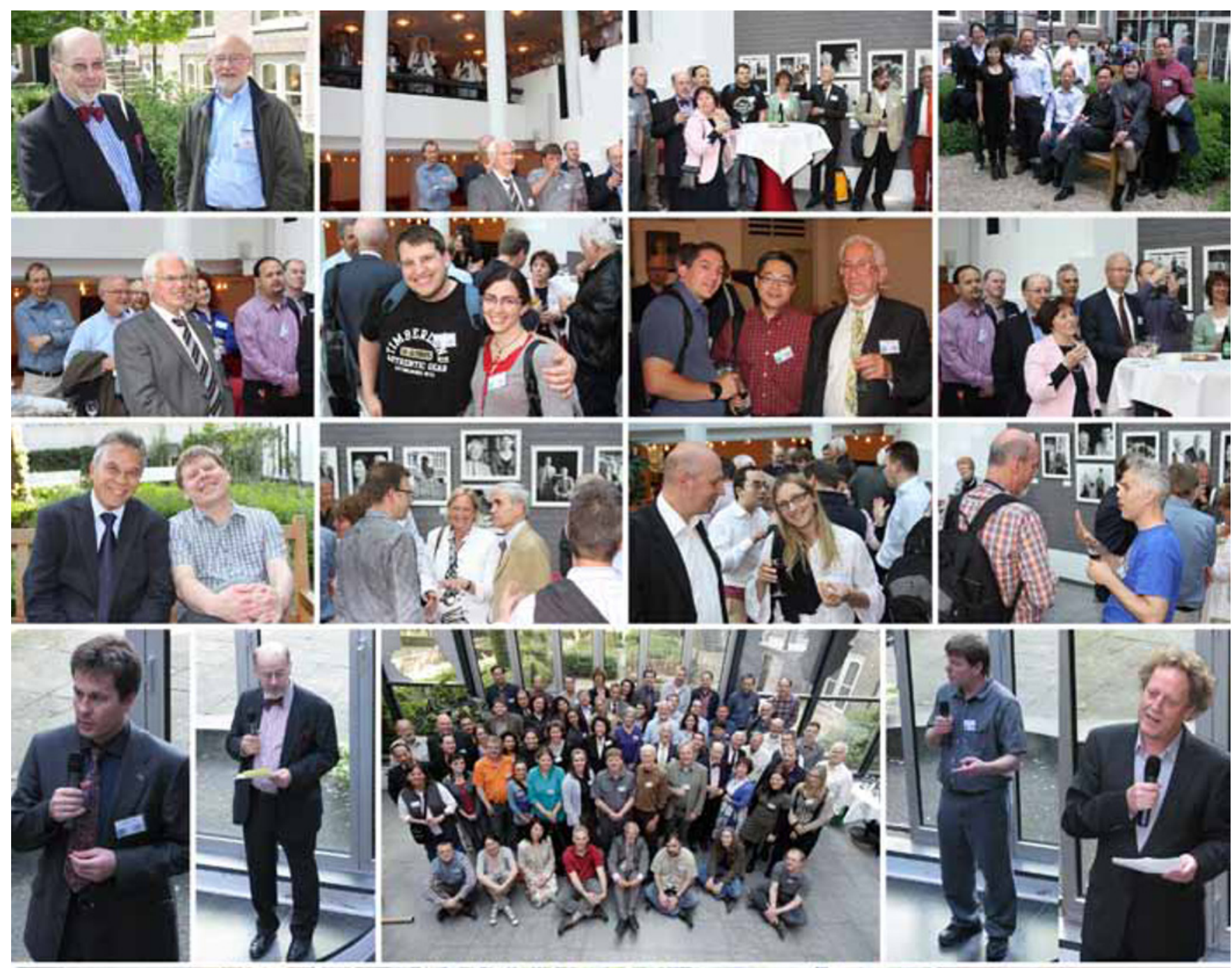

Hoing

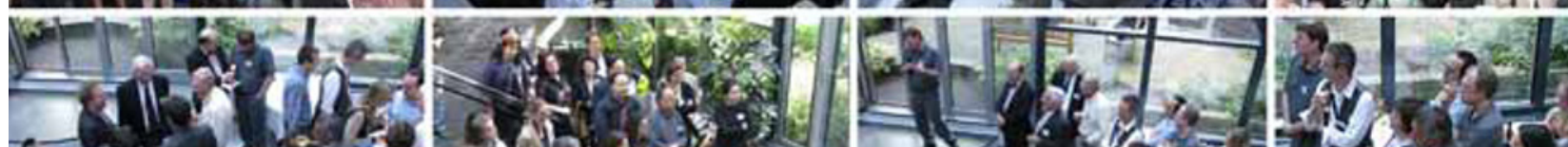
un

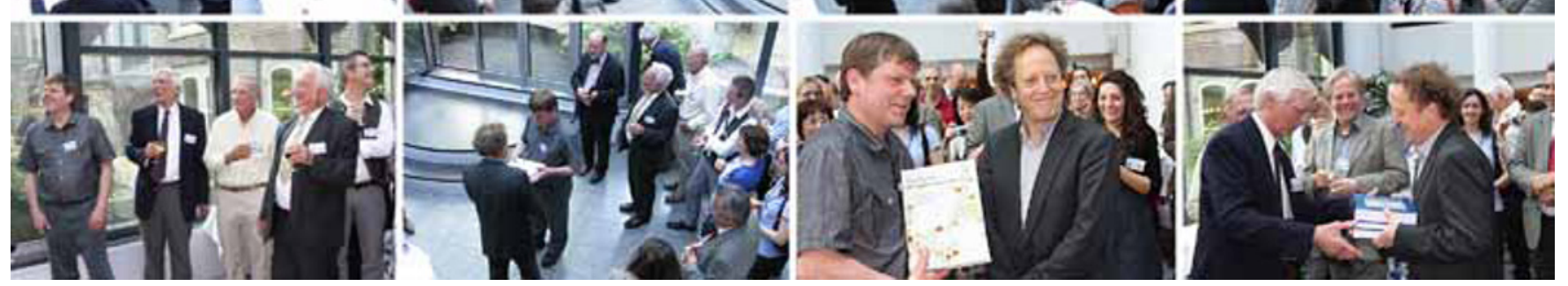

Scenes from the double mycological celebration in the Trippenhuis, headquarters of the Royal Netherlands Academy of Arts and Sciences, Amsterdam, on 20 April 2011: 120 years service by Sybren de Hoog, Robert A Samson, and Joost A Stalpers at the CBS-KNAW Fungal Biodiversity Centre, and the launch and presentation of The Genera of Hyphomycetes and The Yeasts to Theo Mulder, Science Director of the Academy. 